\section{Verzögertes Erbrechen bleibt ein Problem}

\author{
Übelkeit und Erbrechen begleiten viele Chemotherapien, sodass eine \\ Prophylaxe abhängig vom emetogenen Potenzial der Behandlung wichtig ist. \\ Maurice van der Vorst und Kollegen betrachteten die verzögerte Phase bei \\ Anthrazyklin- und Cyclophosphamid-freien moderat emetogenen Chemo- \\ therapieregimen (non-AC MEC).
}

\begin{abstract}
A uch bei moderat emetogenen Chemotherapieregimen (MEC) ohne Anthrazykline oder Cyclosphosphamid kommt es zu chemotherapiebedingter Übelkeit und Erbrechen (CINV). Differenziert wird zwischen der akuten $(<24 \mathrm{~h})$ und verzögerten Phase $(\geq 24 \mathrm{~h})$. $\mathrm{Zu}$ MEC zählen u.a. Oxaliplatin, Irinotecan oder Carboplatin. Aktuelle Leitlinien empfehlen bei non-AC MEC zur Prophylaxe des akuten Erbrechens Palonosetron und Dexamethason, mit Fortsetzung der Dexamethasongabe über mehrere Tage, um verzögertes Erbrechen zu verhindern.

Van der Vorst und Kollegen screenten 247 Publikationen. 9 erfüllten das von ihnen gesetzte Kriterium: eine randomisierte, kontrollierte Studie, in der pros-
\end{abstract}

pektiv die Effizienz von $\geq 2$ antiemetischen Therapien zur Prophylaxe des verzögerten Erbrechens nach non-AC MEC getestet wurde. Endpunkte waren: komplettes Ansprechen, komplette Kontrolle, keine Übelkeit oder Erbrechen sowie die Notwendigkeit einer Rescue-Medikation.

Eingesetzt wurden Palonosetron, Aprepitant, Casopitant, die fixe Kombination von Netupitant und Palonosetron (NEPA), Olanzapin und Megestrolazetat. Die Heterogenität der Studien limitiert nach Ansicht der Forscher die Vergleichsmöglichkeiten. Auch wurden AC und non-AC-MEC-Regimes in Subgruppenanalysen oft miteinander kombiniert, weshalb sich nur schwer Schlüsse für nonAC MEC ziehen ließen. In zwei Studien konnte keine Überlegenheit von Palono- setron gegenüber $5-\mathrm{HT}_{3}$-Rezeptorantagonisten $\left(5-\mathrm{HT}_{3}-\mathrm{RA}\right)$ der ersten Generation bei der Prophylaxe des akuten und verzögerten Erbrechens nachgewiesen werden. Die Zugabe eines $\mathrm{NK}_{1}$-Rezeptorantagonisten zu einem $5-\mathrm{HT}_{3}-\mathrm{RA}$ der ersten $\mathrm{Ge}$ neration verringerte die Inzidenz des verzögerten Erbrechens nicht signifikant. Bei der Kombination von Dexamethason mit Palonosetron war es egal, ob Dexamethason an einem oder mehreren aufeinanderfolgenden Tagen verabreicht wurde. NEPA, Olanzapin und Megestrolazetat erwiesen sich als hoch effizient mit hohen Raten kompletter Response.

Fazit: Weitere Anstrengungen erscheinen notwendig, um die Effizienz der antiemetischen Prophylaxe zu verbessern und insbesondere Dexamethason-bedingte Nebenwirkungen zu vermeiden.

Kathrin von Kieseritzky

Van der Vorst MJ et al. Prophylactic treatment for delayed chemotherapy-induced nausea and vomiting after non-AC based moderately emetogenic chemotherapy: a systematic review of randomized controlled trials. Support Care Cancer. 2015;23(8):2499-506.

\title{
Daueranwendung von intranasalem Fentanyl
}

\section{Etwa jeder zweite Krebspatient erlebt Durchbruchschmerzen. Sie beeinträchti- gen die Lebensqualität und die Teilhabe am beruflichen und sozialen Leben. Intranasales Fentanyl dauerhaft anwenden zu können, wäre ein großer Gewinn.}

Ty ypisch für Durchbruchschmerzen sind plötzliches Einsetzen, kurze Dauer und starke Intensität der Schmerzen. Bei Erwachsenen, die gegen chronische Tumorschmerzen eine Dauerbehandlung mit Opioiden erhalten, hat sich intranasales Fentanyl (INFS) bewährt. Nun wurde in einer 6-monatigen prospektiven Kohortenstudie überprüft, wie effizient und langfristig verträglich es bei opioidtoleranten Patienten mit fortgeschrittener maligner Erkrankung ist.

Die erwachsenen Patienten waren mit einer fixen, oralen, opioidbasierten $\mathrm{Ba}$ sistherapie schmerztherapeutisch eingestellt. Die Schmerzintensität lag damit unter 4 einer numerischen Skala von 0-10. Zu Beginn der Behandlung erleb- ten die Patienten 1-3 Episoden von Durchbruchschmerzen pro Tag, ihre geschätzte Lebenserwartung lag über 6 Monaten. $\mathrm{Zu}$ Beginn und in 4-WochenIntervallen wurde über 6 Monate hinweg die Effizienz der INFS-Therapie beurteilt. Nebenwirkungen beim Langzeitgebrauch, Schlafqualität, Abbruchrate und die Gründe dafür wurden dokumentiert.

Von den ursprünglich 75 befragten Patienten lagen bei 34 (45,3\%) Followup-Daten von 3 Monaten vor, bei 12 (16\%) Follow-up-Daten von 6 Monaten. Die mittlere Opioiddosis reichte von 111-180 mg Morphin-Äquivalenten pro Tag, die mittlere INFS-Dosis von 87-119 $\mu \mathrm{g}$ pro Tag. Die angewendeten Dosen an Opioiden und INFS stiegen mit der Zeit.
Nebenwirkungen wie Kopfschmerzen, Benommenheit, Schwindel und Verstopfung waren selten und meist der Wechselwirkung mit den Opioiden geschuldet, ihre Intensität meist mild, bei einigen Patienten moderat. Während der ersten 3-4 Monate verbesserte sich die Schlafqualität signifikant. Die meisten Patienten erlebten die Wirksamkeit von INFS gegen die Durchbruchschmerzen als gut bis exzellent. Die Effizienz nahm bis zum 3. Monat zu. Abbrüche wegen Nebenwirkungen gab es nicht.

Fazit: Die Langzeitanwendung von INFS zur Behandlung von Durchbruchschmerzen bei fortgeschrittenen Tumorleiden war sicher und effizient und ohne schwerwiegende Nebenwirkungen während des 6-monatigen Gebrauchs. Die Therapie wirkte sich positiv auf die Schlafqualität aus. Kathrin von Kieseritzky

Mercadante $S$ et al. Long-term efficacy and tolerability of intranasal fentanyl in the treatment of breakthrough cancer pain. Support Care Cancer. 2015;23(5):1349-54. 